University of South Florida

DIGITAL COMMONS

Digital Commons @ University of

@ UNIVERSITY OF SOUTH FLORIDA

South Florida

Rehabilitation and Mental Health Counseling

Faculty Publications

Rehabilitation and Mental Health Counseling

2010

\title{
Elder Path Tortuosity Covaries with MMSE Geographical \\ Orientation Subscale
}

William D. Kearns

University of South Florida, kearns@usf.edu

Follow this and additional works at: https://digitalcommons.usf.edu/mhs_facpub

\section{Scholar Commons Citation}

Kearns, William D., "Elder Path Tortuosity Covaries with MMSE Geographical Orientation Subscale" (2010). Rehabilitation and Mental Health Counseling Faculty Publications. 40.

https://digitalcommons.usf.edu/mhs_facpub/40

This Article is brought to you for free and open access by the Rehabilitation and Mental Health Counseling at Digital Commons @ University of South Florida. It has been accepted for inclusion in Rehabilitation and Mental Health Counseling Faculty Publications by an authorized administrator of Digital Commons @ University of South Florida. For more information, please contact digitalcommons@usf.edu. 


\section{Activity monitoring}

C.G. WILLEMS (Convener). The use of activity monitoring in care for persons with dementia. Gerontechnology 2010;9(2):175; doi:10.4017/gt.2010.09.02.018.00 Participants: W.D. KEARNS (USA), M.D. SPREEUWENBERG (NETHERLANDS), C.G.M.H. WILLEMS (NETHERLANDS), and L.L.P. DE WITTE (NETHERLANDS). ISSUE Dementia is characterized as a disease with a loss of orientation to time, place and person, which generally leads to a gradual loss of the capacity to perform activities of daily life in an independent way and a growing dependence of care support. CONTENT Ideally, technology can provide a caregiver with information on the activities performed by the care receiver without an undesired disturbance of the activity itself. What requirements does this have for the development process? What does it mean for user-centered product design methods? How can these technologies be targeted to the specific requirements of the system of use? What specific issues have to be taken into account to successfully implement the technologies presented that are aimed at giving timely information to formal and informal care providers? STRUCTURE The work of Brul presented by Willems will describe a multi-step framework using an evolving set of scenarios to start the development process. Functional requirements (FR) and non-functional requirements (NFR) are defined using caregivers evaluation of these scenarios. Together, the FR and NFR are used to support the development of a system architecture that will be used in the technology development process. Kearns will follow using activity measurement in gait analysis in persons with age-related cognitive decline. Measurements are done in both a laboratory setting and a real life environment. The difference of environment leads to a difference in the detection of gait disturbances and can be of therapeutic relevance. The work of Neyens presented by de Witte aims to develop and evaluate an innovative system for monitoring physical frailty indicators (weight, balance, gait, muscle strength, endurance and physical activity) in community-dwelling older people, which will give direct feedback to the user. Spreeuwenberg will describe the implementation of an activity monitoring system that is available commercially as a part of the informal and formal care provision to people with dementia living at home in the Netherlands. This report deals with the (introduction of) changes in the care routines as supported by the use of this technology. The report demonstrates that the implementation requires a structured effort in which the technology submerges in every day practice. CONCLUSION These presentations outline an approach that enables the development and implementation of ambient technology that starts by measuring human activity in an unobtrusive way. Thereby enabling the person with dementia to continue every day routines as long as possible. It also stresses the potential of measuring activity in an everyday living situation and incorporate this procedure in care delivery processes.

Keywords: human activity measurement, dementia, support of care provision

Address: Saxion University, M.H.Tromplaan 28, 7513AB Enschede, Netherlands; E: c.g.m.h.willems@saxion.nl

M.A. BRUL, R.M.H.A. HUIS in 'T VELD, J.E.W.C. van GeMERT-PIJnEN, C.G.M.H. Willems, H.J. HERMENS. Designing a telecare system for caregivers: A user-centered approach using scenarios. Gerontechnology 2010;9(2):175-176; doi:10.4017/gt.2010.09.02.019.00 Purpose Telecare systems show the potential for elderly to remain in-home for a longer period, by increasing early detection of behavioural changes and adapting home care delivery. As $37 \%$ of the tele- 
care initiatives fail due to a lack of user acceptance of the technology ${ }^{1}$, involving users in the design process and taking their needs as onset for design is regarded as a prerequisite. However, involving caregivers as contemplated users in the design process raises several barriers. Caregivers are overall technically illiterate, unable to address their needs for technology and their involvement faces a vocabulary gap with developers ${ }^{2}$. Secondly, the fuzzy process of needs elicitation and translating needs into formal requirements is part of the challenge to overcome with this mutual vocabulary gap. Our work presents a practical, multi-step framework to involve caregivers in the initial phase of a design process of a telecare system. The framework is deployed to design conceptually an activity monitoring system to support informal and professional caregivers in home care delivery for solitary, in-home, demented persons. Method A multi-step framework was deployed based on contextual design and user centered design methods, supported by an evolving set of scenarios ${ }^{3}$. Based on a contextual study of in-home care delivery, a user story was written and used as onset for eliciting caregivers' needs in a workshop. Secondly, functional requirements (FRs) and non-functional requirements (NFRs) were defined based on caregivers' evaluation of a conceptual scenario and mock-ups that were generated based on the insights from the workshop. Results \& Discussion In the three hour workshop the user story lead to a clear discussion and prioritization of needs, where caregivers $(n=9)$ underlined the need for technology to improve the quality of home care delivery. The two most important activities, eating and drinking and sleeping, were selected by the group for further development. The conceptual scenario and mock-ups were insightful for caregivers $(n=5)$ and they were able to constructively evaluate and (re)define a set of 28 FRs and 7 NFRs. The FRs were defined qualitatively and reflected the functionality of the system in terms of specific activities to monitor generic data transmission functions and method of presenting the information (interface). The NFRs were often articulated as concerns regarding the contemplated system and reflected practical concerns for the demented persons (for instance, non-wearable sensors, unobtrusiveness) and the caregivers' work activities (for instance, ease of use, validity of information). The NFRs are used as design criteria to make design choices in the following step towards a system architecture. This study showed that scenarios enabled us to elicit unarticulated needs of caregivers and evaluate FRs and NFRs. Secondly, scenarios enabled caregivers to understand and respond to technical concepts in order to involve them constructively in the design process. A future challenge is to translate needs to requirements in a workshop involving both caregivers and technicians in order to define requirements quantitatively, using scenarios as a common platform for discussion.

\section{References}

1. Broens THF, Huis in't Veld RMHA, Vollenbroek-Hutten MMR, Hermens HJ, Halteren AT van, Nieuwenhuis LJM. Determinants of successful telemedicine implementations: a literature study. Journal of Telemedicine and Telecare 2007;13(6):303-309

2. Scandurra I, Hägglund M, Koch S. From user needs to system specification: Multi-disciplinary thematic seminars as a collaborative design method for development of health information systems. Journal of Biomedical Informatics 2008;41(4):557-569

3. Beynon-Davies $P$, Holmes S. Design breakdowns, scenarios and rapid application development. Information and Software Technology 2002;44(10):579-592

Keywords: needs elicitation, scenarios, user centered design

Address: Saxion University of Applied Sciences, Enschede, Netherlands;

E: m.a.brul@saxion.nl

W.D. KEARNS. Elder path tortuosity covaries with MMSE geographical orientation subscale. Gerontechnology 2010;9(2):176-177; doi:10.4017/gt.2010.09.02.020.00 Purpose Research using traditional measures of gait and balance suggests that performance is linked to age-related cognitive decline ${ }^{1}$. Using Ultra Wideband RFID transponders we have demonstrated that, outside the testing environment, variation in locomotion, which results in more tortuous paths, is associated with Mini Mental State Exam (MMSE) measured cognitive decline ${ }^{2}$. We hypothesized that the ability to orient oneself in space and time, as measured by the 'Geographical Orientation' and 'Temporal Orientation' MMSE subscales, would be inversely related to the tortuosity in elders' paths recorded in their home environments, which may possibly reflect their positional uncertainty within familiar surroundings. Method Daytime locomotion by 25 


\section{Activity monitoring}

elders (19 female; 14 with a clinical diagnosis of dementia; average age 86.2) were monitored in common living areas of two ALFs for 30 days using miniature radio transponders that measured participant location to $20 \mathrm{~cm}$ at $0.4 \mathrm{~s}$ intervals. The MMSE and Revised Algase Wandering ScaleCommunity Version (RAWS-CV) were administered following the 30 day observation period. Results \& Discussion Fractal Dimension, a measure of locomotor spatial path variability, correlated negatively and significantly with the overall MMSE ( $r=-0.44, n=25$, $\mathrm{p}<0.05$ ) but only the Geographical Orientation $(-0.66, n=25, p<0.001)$ and Temporal Orientation $(r=-0.39, n=25$, $p=0.055$ ) subscales of the MMSE

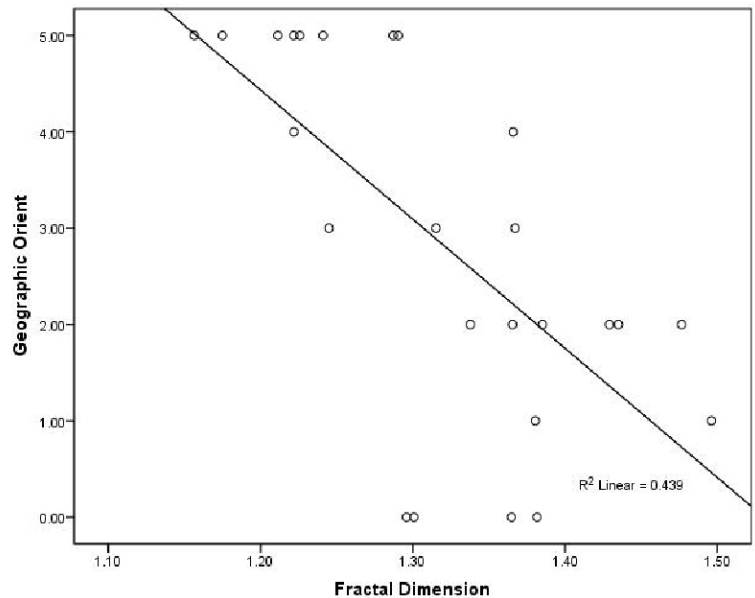

Figure 1. Relationship of the MMSE Geographical Orientation subscale to path variability as measured by fractal dimension were significantly correlated with Fractal Dimension. Fractal Dimension levels trended higher, but were not significantly higher, for persons identified as wanderers by the RAWS-CV ( $F=3.69, M S=0.028, d f=1,23, p=0.07)$. Conclusions Tortuosity is most closely associated with deficits in geographical orientation and temporal orientation as measured by the MMSE. Elders who have difficulties determining their location in familiar environments may produce more tortuous paths as a result of repeated attempts to determine their position. Tortuosity is a linearly distributed continuum against which cognitive function may be plotted. Path monitoring may be a means for early detection of individuals in early cognitive decline, allowing downward trajectories to be therapeutically intercepted at an earlier point where medication can slow or arrest the deterioration before significant CNS damage occurs.

\section{References}

1. Sheridan PL, Hausdorff JM. The Role of Higher-Level Cognitive Function in Gait: Executive Dysfunction Contributes to Fall Risk in Alzheimer's Disease. Dementia and Geriatric Cognitive Disorders 2007;24(2):125-137

2. Kearns W, Nams V, Fozard J. Wireless Fractal Estimation of Tortuosity in Movement Paths Related to Cognitive Impairment in Assisted Living Facility Residents. Methods of Information in Medicine; in Press

Keywords: cognitive impairment, path tortuosity, fractal dimension

Address: Department of Aging \& Mental Health Disparities, Florida Mental Health Institute, College of Behavioral \& Community Sciences, University of South Florida, $13301 \mathrm{~N}$. Bruce B. Downs Blvd. Tampa, Florida 33612, USA; E: kearns@fmhi.usf.edu

J.C.L. Neyens, J. Vermeulen, M.D. Spreeuwenberg, E. van Rossum, D. Hewson, L.P. de WITTE. Monitoring of physical frailty in older people: An innovative system supporting selfmanagement and care. Gerontechnology 2010;9(2):177-178; doi:10.4017/gt.2010.09.02.021.00 Purpose The aim of this project is to develop and evaluate an innovative system for monitoring physical frailty indicators (weight, balance, gait, muscle strength, endurance and physical activity) in community-dwelling older people. The system gives feedback to the user about his/her functional status and warns the user and/or caregivers if a change in the indicators occurs. With this feedback, the system supports the user in his/her self-management regarding physical functioning. It also enables monitoring of the development over time. In this way, the negative consequences of frailty can be postponed or prevented and care can be tuned to the needs related to changes in functional status (reactive care). Method The project consists of three parts. Part one concerns the development of the monitoring system, which consists of a bathroom scale measuring weight and balance, a grip ball measuring strength in the 
hand, and an activity monitor measuring physical activity and gait. Part two regards the development of the feedback- and advisory programme that gives simple and easy to understand feedback to the user, with individual advice regarding physical activity, related to personal goals. Elderly people will be intensively involved in the development process. The results will be tested in small scale pilot studies. Part three is an evaluation study of 50 older persons with early stage frailty symptoms. These patients will be approached through the outpatient fall clinic of Orbis Medical Centre. The participating persons will use the system for 12 months, regularly supported by a physiotherapist. Results $\&$ Discussion The study started in January 2010. In the presentation, the technology and the study approach will be presented, and preliminary results will be shown. The study is ongoing. The evaluation will be directed to technical performance of the system, functionality and usability for the target group, and the added value of the system for the care process as perceived by the users, their informal caregivers and professional caregivers. Also, the relation of the data provided by the system and outcome measures of functional status will be studied.

Keywords: monitor, physical, frailty, elderly, self-management

Address: Maastricht University, Netherlands; E: j.neyens@zw.unimaas.nl

C. Willems, M.D. Spreeunenberg, R. ClaAssen. Activity monitoring as part of care delivery to independently living seniors. Gerontechnology 2010;9(2):178-179;

doi:10.4017/gt.2010.09.02.022.00 Purpose Demographic trends in the southern Netherlands predict an increasing aging population in the coming decade. Since there will not be enough health providers available to meet the growing demand of care of the elderly, there is a need to reorganize the Dutch health care system in a more efficient way. In this process, innovative technologies can play an important role. With the Quiet Care system the activities of the elderly living at home are automatically monitored ${ }^{1,2}$. The monitoring is done by placing infrared sensors in the home at specific locations, such as in the bathroom or in the refrigerator. Clearly interpreted information about the general daily living (ADL) of elderly living at home is shown on a secure web site. Based on this information, providers have a better view of what's happening at home and, when necessary, they can provide care in a more reactive way. In the province of Limburg, a transition project has started aimed to apply the Quiet Care system to enable older people to live longer, independently at home, in a responsible and safe manner. Method A pilot study was conducted with 19 frail elderly clients of the home care organization Proteion $^{3,4}$. A regionally based information infrastructure to handle alerts was organized. A questionnaire was used to measure the clients' quality of life and feelings of safety at baseline and after 26 weeks. After this pilot, protocols for implementation and educational material were developed to support organizations in the implementation of the Quiet Care System. Successively, all teams of Proteion will be educated to work with the system and about 100 clients of Proteion will be supported with Quiet Care in the coming year. Large-scale introduction of the Quiet Care system will, however, have an impact on the demand of care and the way that this care is provided. To study the effects of Quiet Care, an evaluation study is being carried out. The quality of life, feelings of safety, loneliness of clients, the feelings of burden of direct caregivers and the job satisfaction of health providers are monitored at baseline, after three months and after half a year of inclusion. The transition of Quiet Care in the region has been supported by organizing a project group consisting of four other home care providers in the region. Results \& Discussion In the pilot study 11 out of 19 clients indicate that their feeling of security has increased by the system. Caregivers indicated that the system had a clear added value and feel that they have a better view on the daily functioning of the clients ${ }^{2-}$

4 . At this moment, two teams of Proteion are educated to work with the system and 44 clients are supported with Quiet Care. This year four other home care providers will implement Quiet Care in their organization. Both the process evaluation of the implementation and the effect study will be completed this year.

\section{References}

1. Glascock AP, Kutzik DM. Behavioral Telemedicine: A New Approach to the Continuous Nonintrusive Monitoring of Activities of Daily Living. Telemedicine Journal 2000;6(1):33-44 


\section{Activity monitoring}

2. Glascock AP, Kutzik DM. The Impact of Behavioral Monitoring Technology on the provision of Health Care in the Home. Journal of Universal Computer Science 2006;12(1):59-79

3. Willems C. Caring Home.nl: Verslag van een pilot uitgevoerd bij Proteion Thuis [in Dutch]. Utrecht: Vilans; 2008

4. Willems C, Rietman J, Glascock AP, Kutzik DM. The Role of an Informatics-Based Telehomecare in a Person-Centered Service Delivery Model: Findings from a Pilot Study in the Netherlands; 2008; not published, available from the author

Keywords: home care, activity monitoring, frail elderly, quiet care

Address: Centre of Research Technology in Care, Zuyd University, Heerlen, Netherlands;

E: c.g.m.h.willems@hszuyd.nl 